\title{
Induced graphs of uniform spanning forests
}

\author{
Russell Lyons* $^{*} \quad$ Yuval Peres ${ }^{\dagger} \quad$ Xin Sun ${ }^{\ddagger}$
}

\begin{abstract}
Given a subgraph $H$ of a graph $G$, the induced graph of $H$ is the largest subgraph of $G$ whose vertex set is the same as that of $H$. Our paper concerns the induced graphs of the components of $\operatorname{WSF}(G)$, the wired uniform spanning forest on $G$, and, to a lesser extent, $\operatorname{FSF}(G)$, the free uniform spanning forest. We show that the induced graph of each component of $\operatorname{WSF}\left(\mathbb{Z}^{d}\right)$ is almost surely recurrent when $d \geq 8$. Moreover, the effective resistance between two points on the ray of the tree to infinity within a component grows linearly when $d \geq 9$. For any vertex-transitive graph $G$, we establish the following resampling property: Given a vertex $o$ in $G$, let $\mathcal{T}_{o}$ be the component of WSF $(G)$ containing $o$ and $\overline{\mathcal{T}_{o}}$ be its induced graph. Conditioned on $\overline{\mathcal{T}_{o}}$, the tree $\mathcal{T}_{o}$ is distributed as $\operatorname{WSF}\left(\overline{\mathcal{T}_{o}}\right)$. For any graph $G$, we also show that if $\mathcal{T}_{o}$ is the component of $\operatorname{FSF}(G)$ containing $o$ and $\overline{\mathcal{T}_{o}}$ is its induced graph, then conditioned on $\overline{\mathcal{T}_{o}}$, the tree $\mathcal{T}_{o}$ is distributed as $\operatorname{FSF}\left(\overline{\mathcal{T}_{o}}\right)$.
\end{abstract}

\section{Résumé}

Étant donné un sous-graphe $H$ d'un graphe $G$, le graphe induit de $H$ est le plus grand sous-graphe de $G$ dont l'ensemble de sommets est le même que celui de $H$. Notre article concerne les graphes induits des composants connexes de $\operatorname{WSF}(G)$, la forêt recouvrante uniforme câblée sur $G$, et, dans une moindre mesure, $\operatorname{FSF}(G)$, la forêt recouvrante uniforme libre. Nous montrons que le graphe induit de chaque composant de $\operatorname{WSF}\left(\mathbb{Z}^{d}\right)$ est presque sûrement récurrent lorsque $d \geq 8$. De plus, la résistance effective entre deux points du rayon de l'arbre à l'infini au sein d'un composant croît linéairement lorsque $d \geq 9$. Pour tout graphe transitif à sommets $G$, nous établissons la propriété de rééchantillonnage suivante: Étant donné un sommet $o$ dans $G$, soit $\mathcal{T}_{o}$ le composant de $\operatorname{WSF}(G)$ qui contient $o$ et $\overline{\mathcal{T}_{o}}$ son graphe induit. Conditionné sur $\overline{\mathcal{T}_{o}}$, l'arbre $\mathcal{T}_{o}$ est distribué comme $\operatorname{WSF}\left(\overline{\mathcal{T}_{o}}\right)$. Pour tout graphe $G$, nous montrons également que si $\mathcal{T}_{o}$ est le composant de $\operatorname{FSF}(G)$ qui contient $o$ et $\overline{\mathcal{T}_{o}}$ est son graphe induit, alors conditionné sur $\overline{\mathcal{T}_{o}}$, l'arbre $\mathcal{T}_{o}$ est distribué comme $\operatorname{FSF}\left(\overline{\mathcal{T}_{o}}\right)$.

Keywords - resampling, recurrence, effective resistance, loop-erased random walk

*Department of Mathematics, Indiana University. Partially supported by the National Science Foundation under grants DMS-1007244 and DMS-1612363 and by Microsoft Research. Email: rdlyons@indiana.edu.

$\dagger$ Kent State University, Kent, OH. Email: yuval@yuvalperes.com.

†Department of Mathematics, Columbia University. Partially supported by Simons Society of Fellows, by NSF Award DMS-1811092, and by Microsoft Research. Email: xinsun@math.columbia.edu. 


\section{Introduction}

Given a finite, connected graph $G$, the uniform spanning tree (UST) on $G$, which we denote by $\operatorname{UST}(G)$, is the uniform measure on the set of spanning trees of $G$. Given a (locally finite) infinite, connected graph $G$, notions of "uniform spanning tree" can be defined via limiting procedures. Suppose $\left\langle G_{n}\right\rangle$ is a sequence of finite, connected subgraphs of $G$. We call $\left\langle G_{n}\right\rangle$ an exhaustion of $G$ if $G_{n} \subset G_{n+1}$ and $\bigcup G_{n}=G$. According to [Pem91], given an exhaustion $\left\langle G_{n}\right\rangle$ of $G$ and a fixed, finite subgraph $H$ of $G$, the weak limit of UST $\left(G_{n}\right) \cap H$ exists. Varying $H$, one obtains a probability measure on subgraphs of $G$, which is called the free spanning forest (FSF) of $G$ and denoted by $\operatorname{FSF}(G)$. On the other hand, for any finite, connected subgraph $H$ of $G$, let $\widehat{H}$ be the graph obtained by identifying all vertices not in $H$ to a single vertex. We call UST $(\widehat{H})$ the wired spanning forest (WSF) of $H$ (relative to $G$ ), which we denote by $\operatorname{WSF}(H)$. Given an exhaustion $\left\langle G_{n}\right\rangle$ of $G$ consisting of induced subgraphs and a fixed, finite subgraph $H$ of $G$, the weak limit of $\operatorname{WSF}\left(G_{n}\right) \cap H$ exists. Varying $H$, one obtains a probability measure on subgraphs of $G$, which is called the wired spanning forest of $G$ and denoted by $\operatorname{WSF}(G)$.

Neither WSF and FSF can have no cycles, but both can have more than one (connected) component. This justifies the notion of spanning forest. UST and its infinite-volume extensions have been an important object in probability and mathematical physics for the last three decades. See [BLPS01, LP16] for a basic reference for some of the theory. See [Sch00, LSW04, Dub09, She16, Sun19] for the important role that UST played in the recent development in two dimensional statistical physics and Schramm-Loewner evolution.

For infinite graphs, WSF is much better understood than FSF. In [Wil96], David Wilson provided an efficient algorithm to sample UST on finite graphs. It was soon extended to sample WSF on infinite graphs [BLPS01] (see also Section 3). This powerful tool allows one to study WSF directly via simple random walk. In particular, it is proved in [BLPS01] that $\operatorname{WSF}(G)$ is concentrated on the set of forests with a unique component if and only if two simple random walks on $G$ intersect a.s. In contrast, there is no known simple condition to determine whether an FSF has a unique component. If $G$ is a Cayley graph of the $d$-dimensional integer lattice $\mathbb{Z}^{d}$ for $d \in \mathbb{N}$, then $\operatorname{WSF}\left(\mathbb{Z}^{d}\right)$ and $\operatorname{FSF}\left(\mathbb{Z}^{d}\right)$ coincide. Moreover, it was shown in $[\mathrm{Pem} 91]$ that $\operatorname{WSF}\left(\mathbb{Z}^{d}\right)$ has a unique component when $1 \leq d \leq 4$ and infinitely many components when $d \geq 5$. In the latter case, the collections of components exhibit an intriguing geometry [BKPS04, HP19].

If $H$ is a subgraph of a graph $G$, including the case that $H$ may have no edges, then the induced subgraph determined by $H$ is the largest subgraph of $G$ whose vertex set is the same as that of $H$. If $H$ is a subgraph of a graph $G$, we define the induced-component graph $\bar{H}$ of $H$ to be the largest subgraph of $G$ whose vertex set is the same as that of $H$ and that has the same connected components as $H$. Thus, an edge of $G$ belongs to $\bar{H}$ if and only if both its endpoints belong to the same component of $H$. We also have that $\bar{H}$ is the union of the induced subgraphs determined by the components of $H$.

Before stating our main results, we make the following conventions throughout the paper. We will use WSF, FSF, UST to denote either probability measures or their samples as long as it is clear from the context what we are referring to. When there is a risk of ambiguity, we use $\operatorname{UST}(G), \operatorname{FSF}(G), \operatorname{WSF}(G)$ to represent probability measures and $\mathfrak{T}(G), \mathfrak{F}_{\mathrm{f}}(G), \mathfrak{F}_{\mathrm{W}}(G)$ to represent their corresponding samples. Similarly, we write $\overline{\operatorname{WSF}(G)}$ for either the law of $\overline{\mathfrak{F}_{\mathrm{w}}(G)}$ or for its sample, $\overline{\mathfrak{F}_{\mathrm{w}}(G)}$, and likewise for the free versions.

The main object of interest in this paper is $\overline{\mathrm{WSF}\left(\mathbb{Z}^{d}\right)}$, which reflects the geometry of $\mathrm{WSF}\left(\mathbb{Z}^{d}\right)$ as a subgraph embedded in $\mathbb{Z}^{d}$. Since $\overline{\operatorname{WSF}\left(\mathbb{Z}^{d}\right)}=\mathbb{Z}^{d}$ when $1 \leq d \leq 4$, the only interesting case is when $d \geq 5$. On the one hand, components of $\overline{\operatorname{WSF}\left(\mathbb{Z}^{d}\right)}$ have stochastic dimension 4 for all $d \geq 5$ 
[BKPS04]. On the other hand, Morris [Mor03] proved that for every graph $G$, simple random walk on each component of $\operatorname{WSF}(G)$ is a.s. recurrent. This leads to the intriguing question of whether the components of $\overline{\mathrm{WSF}\left(\mathbb{Z}^{d}\right)}$ are recurrent or transient.

Theorem 1.1. If $d \geq 8$, almost surely each connected component of $\overline{\mathrm{WSF}\left(\mathbb{Z}^{d}\right)}$ is recurrent.

For a graph $G=(V, E)$, let $f$ be a real function from $V$ to $\mathbb{R}$, and let

$$
\mathcal{E}(f):=\frac{1}{2} \sum_{x, y \in G ; x \sim y}(f(x)-f(y))^{2},
$$

where $x \sim y$ means $x$ and $y$ are adjacent in $G$. Given two disjoint subsets $A$ and $B$ of $V$, the effective resistance between $A$ and $B$ is defined by

$$
\mathrm{R}_{\text {eff }}^{G}(A, B):=\left(\inf \left\{\mathcal{E}(f) ;\left.f\right|_{A}=1,\left.f\right|_{B}=0\right\}\right)^{-1} .
$$

Definition 1.2. An end of a tree is an equivalence classes of infinite simple paths in the tree, where two paths are equivalent if their symmetric difference is finite. Given a vertex $v$ in a forest, write $T_{v}$ for the component of the forest that contains $v$. If $T_{v}$ has one end, then write $\operatorname{Ray}_{v}=\left\langle\operatorname{Ray}_{v}(n)\right\rangle_{n \geq 0}$ for the unique infinite, simple path in $T_{v}$ starting at $v$. Given $v \in \mathbb{Z}^{d}$, let $\mathcal{T}_{v}$ be the component of $\operatorname{WSF}\left(\mathbb{Z}^{d}\right)$ containing $v$.

As discussed following Corollary 1.7, every tree $\mathcal{T}_{v}$ has one end a.s.

Theorem 1.3. Let $v \in \mathbb{Z}^{d}$. If $d \geq 9$, then $\liminf _{n \rightarrow \infty} n^{-1} \mathrm{R}_{\text {eff }}^{\overline{\mathcal{T}_{v}}}\left(v\right.$, $\left.\operatorname{Ray}_{v}(n)\right)>0$ almost surely.

Since $\mathrm{R}_{\text {eff }}^{\overline{\mathcal{T}_{v}}}\left(v, \operatorname{Ray}_{v}(n)\right) \leq n$, Theorem 1.3 means $\mathrm{R}_{\text {eff }}^{\overline{\mathcal{T}_{v}}}\left(v, \operatorname{Ray}_{v}(n)\right)$ grows linearly.

Theorems 1.1 and 1.3 leave open the natural questions whether components of $\overline{\operatorname{WSF}\left(\mathbb{Z}^{d}\right)}$ are recurrent or transient for $d=5,6,7$ and what the growth rate of $\mathrm{R}_{\text {eff }}^{\overline{\mathcal{T}}_{v}}\left(v, \operatorname{Ray}_{v}(n)\right)$ is for $d=5,6,7,8$. Although we do not address those problems here, we prove the following resampling property of $\operatorname{WSF}\left(\mathbb{Z}^{d}\right)$ for all dimensions, which has implications for the behavior of random walks on the components of $\overline{\mathrm{WSF}\left(\mathbb{Z}^{d}\right)}$.

We will use the following notion. Given a graph $G$, let $H$ be a random subgraph of $G$ whose components are infinite graphs. We write $\operatorname{WSF}(H)$ for the unconditional law of the random subgraph of $G$ obtained by first sampling $H$ and then sampling a WSF independently on each component of this instance of $H$. We similarly define $\operatorname{FSF}(H)$.

Theorem 1.4. For all $d \in \mathbb{N}, \operatorname{WSF}\left(\overline{\mathfrak{F}_{\mathrm{w}}\left(\mathbb{Z}^{d}\right)}\right)=\operatorname{WSF}\left(\mathbb{Z}^{d}\right)$, that is, the two measures agree.

Theorem 1.4 implies that for each $v \in \mathbb{Z}^{d}, \operatorname{WSF}\left(\overline{\mathcal{T}_{v}}\right)$ a.s. has a single component for $\mathcal{T}_{v}$ as in Definition 1.2. Therefore, two independent simple random walks on $\overline{\mathcal{T}_{v}}$ a.s. intersect.

Theorems 1.1 and 1.3 are proved in Section 3 and 4 respectively, using quantitative arguments. A vertex-transitive graph is a graph such that given any two vertices, there exists a graph automorphism mapping one vertex to the other (see Section 3.2). Theorem 1.1 can be extended to all vertex-transitive graphs whose volume growth is at least $r \mapsto r^{8}$, while the argument for Theorem 1.3 works for unimodular vertex-transitive graphs whose volume growth is at least $r \mapsto r^{9}$ (see the definition of unimodular preceding Theorem 4.3).

On the other hand, Theorem 1.4 is a corollary of the following set of general results, which will be proved in Section 5 by qualitative arguments. 
Theorem 1.5. For any locally finite, infinite, connected graph $G$, we have

$$
\operatorname{FSF}\left(\overline{\mathfrak{F}_{\mathrm{w}}(G)}\right)=\operatorname{WSF}(G) \text { and } \operatorname{FSF}\left(\overline{\mathfrak{F}_{\mathrm{f}}(G)}\right)=\operatorname{FSF}(G) \text {. }
$$

In particular, the FSF on each component of $\overline{\mathfrak{F}_{\mathrm{W}}(G)}$ and $\overline{\mathfrak{F}_{\mathrm{f}}(G)}$ has a unique component a.s.

An immediate corollary of Theorem 1.5 is

Corollary 1.6. WSF $\left(\overline{\mathfrak{F}_{\mathrm{W}}(G)}\right)=\operatorname{WSF}(G)$ if and only if each component of $\overline{\mathfrak{F}_{\mathrm{W}}(G)}$ has the property that $\mathrm{FSF}=\mathrm{WSF}$.

Corollary 1.6 implies Theorem 1.4 as follows. Recall $\mathcal{E}$ defined as in (1.1). A necessary and sufficient condition for $\operatorname{FSF}(G)=\operatorname{WSF}(G)$ is that the only harmonic functions $f$ on $G$ with $\mathcal{E}(f)<\infty$ are constant functions [BLPS01]. This is known to be the case when $G$ is transitive and amenable, i.e., $\inf _{K} \# \partial K / \# K=0$, where the infimum is over all finite vertex sets $K$ of $G$. By [BLS99, Theorem 5.5], every amenable transitive graph has the property that each component of every random subgraph with automorphism-invariant law also a.s. has no nonconstant harmonic functions $f$ with $\mathcal{E}(f)<\infty$. This gives Theorem 1.4.

Corollary 1.7. $\operatorname{WSF}\left(\overline{\mathfrak{F}_{\mathrm{W}}(G)}\right)=\operatorname{WSF}(G)$ (resp., $\operatorname{FSF}\left(\overline{\mathfrak{F}_{\mathrm{W}}(G)}\right)=\mathrm{FSF}(G)$ ) if each component of $\mathfrak{F}_{\mathrm{W}}(G)$ (resp., $\mathfrak{F}_{\mathrm{f}}(G)$ ) is one-ended, that is, has a single end a.s.

In [LMS08], it is proved that the one-end property of WSF components holds for all transient vertex-transitive graphs (also see [LP16, Theorem 10.49]). Thus WSF $\left(\overline{\mathfrak{F}_{\mathrm{W}}(G)}\right)=\mathrm{WSF}(G)$ in this case. This in particular gives another proof of Theorem 1.4. For more general results on the one-ended property of FSF and WSF, see [LMS08, Hut18].

Inspired by Morris' aforementioned result that each component of WSF on every graph is a.s. recurrent, we conjecture that $\operatorname{WSF}\left(\overline{\mathfrak{F}_{\mathrm{w}}(G)}\right)=\operatorname{WSF}(G)$ for every locally finite, connected graph $G$ as in Theorem 1.5.

Neither WSF $\left(\overline{\mathfrak{F}_{\mathrm{f}}(G)}\right)=\operatorname{FSF}(G)$ nor WSF $\left(\overline{\mathfrak{F}_{\mathrm{f}}(G)}\right)=\operatorname{WSF}(G)$ holds for all graphs. For counterexamples to the first equality, let $G$ be a tree with the property that $\mathfrak{F}_{\mathrm{w}}(G) \neq G$ a.s. (For example, $G$ could be a regular tree.) Then $\mathfrak{F}_{\mathrm{f}}(G)=G$ a.s. while $\operatorname{WSF}\left(\overline{\mathfrak{F}_{\mathrm{f}}(G)}\right)=\operatorname{WSF}(G)$. A counterexample for the second equality will be given in Section 5.2.

Acknowledgment. We thank Pengfei Tang for assistance in completing the proof of Proposition 2.8 , which simplifies arguments in an earlier version of our paper. We are grateful to the referee for several comments that improved the paper. This work was begun while the third author was an intern in the Theory Group at Microsoft Research, Redmond.

\section{Preliminaries}

\subsection{Basic notations}

The set of positive integers is denoted by $\mathbb{N}$. Given a finite set $A$, we write $\# A$ for the cardinality of $A$. Given two sets $A, B$, their symmetric difference $(A \backslash B) \cup(B \backslash A)$ is denoted by $A \triangle B$. We use the asymptotic notation that two nonnegative functions $f(x)$ and $g(x)$ satisfy $f \lesssim g$ if there exists a constant $C>0$ independent of $x$ such that $f(x) \leq C g(x)$. We write $f \gtrsim g$ if $g \lesssim f$ and write $f \asymp g$ if $f \lesssim g$ and $f \gtrsim g$.

Given a graph $G$, write $V(G)$ and $E(G)$ for the vertex and edge sets of $G$, respectively. When $G=\mathbb{Z}^{d}$ for some $d \in \mathbb{N}$, we write $o$ for its origin. If $v, u \in V(G)$ are adjacent, we write $v \sim u$ 
and write $(u, v)$ for the edge between them. For $v \in V(G)$, let $\operatorname{deg}(v)$ be the degree of $v$, which is the number of vertices adjacent to $v$. In our paper, graphs are assumed to be locally finite, that is, $\operatorname{deg}(v)<\infty$ for every $v \in V(G)$. A graph $H$ is called a subgraph of $G$ if $V(H) \subset V(G)$ and $E(H) \subset E(G)$. If $H$ and $H^{\prime}$ are subgraphs of $G$, we write $E(G) \backslash E(H)$ as $G \backslash H$ and $E(H) \triangle E\left(H^{\prime}\right)$ as $H \triangle H^{\prime}$.

Given a family of probability measures $\left\langle\mu_{t}\right\rangle_{t \in T}$ with index set $T$, a coupling of $\left\langle\mu_{t}\right\rangle_{t \in T}$ is a family of random variables $\left\langle X_{t}\right\rangle_{t \in T}$ on a common probability space such that $X_{t}$ is distributed as $\mu_{t}$ for all $t \in T$. Suppose $A$ and $B$ are two probability measures on the space of subgraphs of a graph $G$. If there is a coupling $(\mathfrak{A}, \mathfrak{B})$ of $(A, B)$ such that $\mathfrak{A} \subset \mathfrak{B}$ a.s., we say that $A$ is stochastically dominated by $B$, written as $A \preccurlyeq B$.

Let $I$ be an interval in $\mathbb{Z}$. Suppose $\mathcal{P}=\left\langle v_{i}\right\rangle_{i \in I}$ is a sequence of vertices in $G$ indexed by $I$ such that $v_{i} \sim v_{i+1}$ whenever $i$ and $i+1$ are both in $I$. Then we call $\mathcal{P}$ a path in $G$. If $v_{i} \neq v_{j}$ as long as $i \neq j$, we say $\mathcal{P}$ is simple. If $I=\{0, \ldots, n\}$, then $\mathcal{P}$ is called a finite path and $|\mathcal{P}|:=n$ is called the length of $\mathcal{P}$. We call the path $\left\langle v_{n-i}\right\rangle_{0 \leq i \leq n}$ the reversal of $\mathcal{P}$. If we further have $v_{0}=v_{n}$, then we call $\mathcal{P}$ a (rooted) loop $^{1}$ and $v_{0}$ the $\operatorname{root}$ of $\mathcal{P}$. If $I=\mathbb{N} \cup\{0\}$ (resp., $I=\mathbb{Z}$ ), we call $\mathcal{P}$ an infinite (resp., bi-infinite) path. We call $t$ a cut time of $\mathcal{P}$ if $\left\{v_{i}\right\}_{i<t} \cap\left\{v_{i}\right\}_{i>t}=\varnothing$.

Given $x, y \in V(G)$, let $d_{G}(x, y)$ be the minimal length of a path starting from $x$ and ending at $y$ if $x, y$ are in the same component of $G$ and $\infty$ otherwise. We call $d_{G}(\cdot, \cdot)$ the graph distance on $G$. For $v \in V(G)$ and $r>0$, let $B_{G}(v, r):=\left\{x \in V(G) ; d_{G}(v, x) \leq r\right\}$. We identify $B_{G}(v, r)$ with its induced subgraph.

A graph is called a forest if for any pair of distinct vertices there exists at most one simple path connecting them. A connected forest is called a tree. Given a connected graph $G$, a spanning tree (resp., forest) on $G$ is a subgraph $T \subset G$ such that $T$ is a tree (resp., forest) and $V(T)=V(G)$.

The simple random walk on $G$ is the Markov chain $\langle S(n)\rangle_{n \geq 0}$ on the state space $V(G)$ such that $\mathbb{P}[S(n+1)=u \mid S(n)=v]=\operatorname{deg}(v)^{-1}$ for all $u \sim v$ and $n \geq 0$. The transition kernel $p$ of $G$ is defined by $p_{t}(x, y)=\mathbb{P}[S(t)=y]$ for $x, y \in V(G)$ and $t \in \mathbb{N} \cup\{0\}$, where $S$ is a simple random walk on $G$ starting from $x$. When $G=\mathbb{Z}^{d}$, it is well known that $p_{t}(o, o) \asymp t^{-d / 2}$ for even $t \geq 2$.

\subsection{Wilson's algorithm}

Given a finite path $\mathcal{P}=\left\langle v_{i}\right\rangle_{0 \leq i \leq n}$ in a graph $G$ of length $n \in \mathbb{N}$, the (forward) loop erasure of $\mathcal{P}$ (denoted by $\mathbf{L E}[\mathcal{P}]$ ) is the path defined by erasing cycles in $\mathcal{P}$ chronologically. More precisely, we define $\mathbf{L E}[\mathcal{P}]$ inductively as follows. The first vertex $u_{0}$ of $\mathbf{L E}[\mathcal{P}]$ equals $v_{0}$. Supposing that $u_{j}$ has been set, let $k$ be the last index such that $v_{k}=u_{j}$. Set $u_{j+1}:=v_{k+1}$ if $k<n$; otherwise, let $\mathbf{L E}[\mathcal{P}]=\left\langle u_{i}\right\rangle_{0 \leq i \leq j}$. If $\mathcal{P}$ is an infinite path that visits no vertex infinitely many times, then we define $\mathbf{L E}[\mathcal{P}]$ in a similar fashion. In particular, if $S$ is a sample of simple random walk on a transient graph $G$, then $\mathbf{L E}[S]$ is defined a.s. In such a case, we call the law of $\mathbf{L E}[S]$ the loop-erased random walk (LERW) on $G$.

In [Wil96], Wilson discovered an algorithm for sampling uniform spanning trees on finite graphs using loop-erased random walk. In [BLPS01], Wilson's algorithm was adapted to sample WSF on a transient graph $G$. This method is called Wilson's algorithm rooted at infinity, which we now review. The algorithm goes by sampling a growing sequence of subgraphs of $G$ as follows. Set $\mathcal{T}_{0}:=\varnothing$. Inductively, for each $n \in \mathbb{N}$, choose $v_{n} \in V(G) \backslash V\left(\mathcal{T}_{n-1}\right)$ and run a simple random walk starting at $v_{n}$. Stop the walk when it hits $\mathcal{T}_{n-1}$ if it does; otherwise, let it run indefinitely. Denote the resulting path by $\mathcal{P}_{n}$, and set $\mathcal{T}_{n}:=\mathcal{T}_{n-1} \cup \mathbf{L E}\left[\mathcal{P}_{n}\right]$. Write $\mathfrak{F}_{\mathrm{w}}:=\bigcup_{n} \mathcal{T}_{n}$. According to

\footnotetext{
${ }^{1}$ This is a topological loop, also called a cycle in graph theory, as opposed to the term "loop" in graph theory.
} 
[BLPS01, Theorem 5.1], no matter how $\left\langle v_{n}\right\rangle_{n \geq 1}$ are chosen, as long as $V\left(\mathfrak{F}_{\mathrm{w}}\right)=V(G)$, the law of $\mathfrak{F}_{\mathrm{w}}$ is $\operatorname{WSF}(G)$.

\subsection{Bounds on effective resistance}

Nash-Williams' inequality (see, e.g., [LP16, Section 2.5]) is a useful lower bound for the effective resistance. Here we record a generalization of Nash-Williams' inequality.

Lemma 2.1. Given a graph $G$ with two disjoint subsets $A$ and $B$ of $V(G)$, a set $\mathcal{C} \subset E(G)$ is called a cut set between $A$ and $B$ if $\forall o \in A$ and $\forall z \in B$, every path from o to $z$ must use an edge in $\mathcal{C}$. Suppose $\mathcal{C}_{1}, \ldots, \mathcal{C}_{n}$ are cut sets between $A$ and $B$ for some $n \in \mathbb{N}$. For $e \in E$, let

$j(e):=\#\left\{k ; e \in \mathcal{C}_{k}\right\}$. Then $\mathrm{R}_{\mathrm{eff}}^{G}(A, B) \geq \sum_{k=1}^{n}\left(\sum_{e \in \mathcal{C}_{k}} j(e) c(e)\right)^{-1}$.

Proof. The proof is the same as the classical case in [LP16, Section 2.5], with a slight modification when applying the Cauchy-Schwarz inequality. We leave the details to the reader.

This is equivalent to having disjoint cutsets in a subdivided network. In that form but for recurrence of infinite, locally finite networks, it was given by [NW59], who also proved that the existence of such cutsets in a subdivided network is necessary for recurrence. See [McG91] for an extension to non-locally finite networks.

The next lemma says that effective resistance is stable under local modification.

Lemma 2.2. Suppose $H$ and $H^{\prime}$ are two connected subgraphs of a graph $G$ such that $\#\left(H \triangle H^{\prime}\right)<$ $\infty$. Then there exists a constant $c>0$ depending on $G, H, H^{\prime}$ such that $\mathrm{R}_{\mathrm{eff}}^{H}(u, v) \leq \mathrm{R}_{\mathrm{eff}}^{H^{\prime}}(u, v)+c$ for all $u, v \in V(H) \cap V\left(H^{\prime}\right)$.

Proof. It suffices to show that if $H^{\prime} \subset H$ and $\#\left(E(H) \backslash E\left(H^{\prime}\right)\right)=1$, then there exists $c^{\prime}>0$ depending only on $H$ and $H^{\prime}$ but not on $u, v \in V\left(H^{\prime}\right)$ such that

$$
0 \leq \mathrm{R}_{\text {eff }}^{H^{\prime}}(u, v)-\mathrm{R}_{\mathrm{eff}}^{H}(u, v) \leq c^{\prime} .
$$

Once this is proved, a similar statement then follows for $H^{\prime} \subset H$ and $\#\left(E(H) \backslash E\left(H^{\prime}\right)\right)<\infty$. Then the general case follows by comparing both $H$ and $H^{\prime}$ to the union graph $H \cup H^{\prime}$ of the two.

By Rayleigh's monotonicity principle (see, e.g., [LP16, Section 2.4]), adding an edge can only decrease the effective resistance, hence $\mathrm{R}_{\text {eff }}^{H^{\prime}}(u, v) \geq \mathrm{R}_{\text {eff }}^{H}(u, v)$. To prove the other direction of (2.1), we use Thomson's principle (see, e.g., [LP16, Section 2.4]) that the effective resistance between two vertices is the minimum energy (i.e., the sum of the squares of all edge flows) among all unit flows between the two vertices. We may start from the minimizing flow for $H$ from $u$ to $v$ and then construct a flow on $H^{\prime}$ between the same vertices by replacing the current flow along the removed edge $e$ with a flow along a path in $H^{\prime}$ connecting the two endpoints of $e$. This increases the flow energy by an additive constant that depends only on $H$ and $H^{\prime}$.

\subsection{Indistinguishability of WSF components}

In this subsection, we review a basic ergodic-theoretic property of components in WSF on transient vertex-transitive graphs. We call a triple $(G, \rho, \omega)$ a subgraph-decorated rooted graph if $G$ is a locally finite, connected graph, $\rho$ is a distinguished vertex in $G$ called the root, and $\omega$ is a function from $E(G)$ to $\{0,1\}$. We think of $\omega$ as a distinguished subgraph spanned by the edges $\{e \in E(G) ; \omega(e)=1\}$. Given two such triples $(G, \rho, \omega)$ and $\left(G^{\prime}, \rho^{\prime}, \omega^{\prime}\right)$, an isomorphism between 
them is a graph isomorphism between $G$ and $G^{\prime}$ that preserves the root and the subgraph. Write $\omega_{\rho, r}$ for the restriction of $\omega$ to $B_{G}(\rho, r)$. Let $\mathcal{G}_{\bullet}^{\{0,1\}}$ be the space of subgraph-decorated rooted graphs modulo isomorphisms. We endow $\mathcal{G}_{\bullet}^{\{0,1\}}$ with the local topology where two elements $(G, \rho, \omega)$ and $\left(G^{\prime}, \rho^{\prime}, \omega^{\prime}\right)$ in $\mathcal{G}_{\bullet}^{\{0,1\}}$ are close if and only if $\left(B_{G}(\rho, r), \rho, \omega_{\rho, r}\right)$ and $\left(B_{G^{\prime}}\left(\rho^{\prime}, r\right), \rho^{\prime}, \omega_{\rho^{\prime}, r}^{\prime}\right)$ are isomorphic to each other for some large $r$.

Given $(G, v, \omega) \in \mathcal{G}_{\bullet}^{\{0,1\}}$, we define $K_{\omega}(v)$ to be the connected component of $v$ in $\omega$. A Borelmeasurable set $\mathcal{A} \subset \mathcal{G}_{\bullet}^{\{0,1\}}$ is called a component property if $(G, v, \omega) \in \mathcal{A}$ implies $(G, u, \omega) \in \mathcal{A}$ for all $u \in K_{\omega}(v)$. Given a component property $\mathcal{A}$, we say that a connected component $K$ of $\omega$ has property $\mathcal{A}$ if $(G, u, \omega) \in \mathcal{A}$ for some (and equivalently every) $u \in V(K)$. A component property $\mathcal{A}$ is called a tail component property if $(G, v, \omega) \in \mathcal{A}$ implies $\left(G, v, \omega^{\prime}\right) \in \mathcal{A}$ for all $\omega^{\prime} \subseteq E(G)$ such that $\omega \triangle \omega^{\prime}$ and $K_{\omega}(v) \triangle K_{\omega^{\prime}}(v)$ are both finite.

As a corollary of [HN17, Theorem 1.20], we have

Lemma 2.3. Suppose $G$ is a transient, vertex-transitive graph. For every tail component property $\mathcal{A}$, either almost surely every connected component of $\operatorname{WSF}(G)$ has property $\mathcal{A}$, or almost surely none of the connected components of $\operatorname{WSF}(G)$ have property $\mathcal{A}$.

By Lemma 2.2, for a vertex-transitive graph, both the properties in Theorems 1.1 and 1.3 are tail component properties. Therefore, we have

Lemma 2.4. Consider $\operatorname{WSF}\left(\mathbb{Z}^{d}\right)$ for $d \geq 5$. Recall the notations $\mathcal{T}_{v}$ and $\operatorname{Ray}_{v}$ in Definition 1.2. Let $E_{v}$ be the event that $\overline{\mathcal{T}}_{v}$ is recurrent and $F_{v}$ be the event $\liminf _{n \rightarrow \infty} n^{-1} \mathrm{R}_{\text {eff }}^{\overline{\mathcal{T}_{v}}}\left(v, \operatorname{Ray}_{v}(n)\right)>0$. Then neither $\mathbb{P}\left[E_{v}\right]$ nor $\mathbb{P}\left[F_{v}\right]$ depends on $v$. Moreover, both $\mathbb{P}\left[E_{v}\right]$ and $\mathbb{P}\left[F_{v}\right]$ belong to $\{0,1\}$. The same holds with $\mathbb{Z}^{d}$ replaced by any transient, vertex-transitive graph.

\subsection{Two-sided random walk and loop-erased random walk}

For $d \in \mathbb{N}$, let $S^{1}$ and $S^{2}$ be two independent simple random walks on $\mathbb{Z}^{d}$ starting from the origin of $\mathbb{Z}^{d}$. For $n \in \mathbb{Z}$, let $S(n):=S_{1}(n)$ if $n \geq 0$ and $S(n):=S^{2}(-n)$ if $n<0$. We call the law of the bi-infinite path $\langle S(n)\rangle_{n \in \mathbb{Z}}$ the two-sided random walk on $\mathbb{Z}^{d}$. It is standard that $\mathbb{P}\left[S_{1}([0, \infty)) \cap S_{2}([1, \infty))=\varnothing\right]>0$ if and only if $d \geq 5$ (see, e.g., [LP16, Theorem 10.24]). For $d \geq 5$, consider the event

$$
E:=\left\{\mathbf{L E}\left[S^{1}\right](m) \neq S^{2}(n) \text { for all } m \geq 0, n \geq 1\right\} .
$$

Since with positive probability 0 is a cut time of $S$, we have that $\mathbb{P}[E]>0$. Define $\widetilde{S}(n)$ to be $\mathbf{L E}\left[S^{2}\right](-n)$ for $n \leq 0$ and $\mathbf{L E}\left[S^{1}\right](n)$ for $n \geq 0$. The conditional law of $\langle\widetilde{S}(n)\rangle_{n \in \mathbb{Z}}$ conditioned on $E$ is called the two-sided loop-erased random walk on $\mathbb{Z}^{d}$. It is clear that without loop-erasures, $\langle S(n+1)-S(n)\rangle_{n \in \mathbb{Z}}$ is stationary and ergodic; indeed, it is an IID sequence. In fact, two-sided LERW also has stationary, ergodic increments:

Lemma 2.5. Suppose $X$ is two-sided loop-erased random walk on $\mathbb{Z}^{d}$ for $d \geq 5$. Then $\langle X(n+1)-$ $X(n)\rangle_{n \in \mathbb{Z}}$ is stationary and ergodic.

Lawler [Law80] introduced the two-sided LERW on $\mathbb{Z}^{d}(d \geq 5)$ and showed that it is the local limit of the usual LERW viewed from nodes with large index. An essential ingredient to the proof of Lemma 2.5 is the reversibility of the loop-erasing operation for simple random walk, which was also first proved in [Law80]. Given the reversibility, we observe that Lemma 2.5 can be deduced from the ergodicity of the two-sided random walk and the following basic fact from ergodic theory (see, e.g., [Pet83]). 
Lemma 2.6 (Kac's Lemma). Suppose $\Omega$ is a measurable space and $T: \Omega \rightarrow \Omega$ is measurable. Suppose $\mathbb{P}$ is a probability measure on $\Omega$ which is preserved by $T$ and is ergodic. Let $A \subset \Omega$ be an event such that $\mathbb{P}[A]>0$ and let $\tau(\omega):=\inf \left\{n \in \mathbb{N} ; T^{n}(\omega) \in A\right\}$ for all $\omega \in \Omega$. Let $T_{A}(\omega):=T^{\tau(\omega)}(\omega)$ for all $\omega \in A$. Then $T_{A}$ is an ergodic, measure-preserving map from $A$ to $A$ under the conditional probability measure $\mathbb{P}[\cdot \mid A]$. Moreover, $\mathbb{E}[\tau \mid A]=\mathbb{P}[A]^{-1}$.

To put Lemma 2.5 in the setting of Lemma 2.6, let us consider the two-sided simple random walk $S$. Since $S$ can be almost surely decomposed into finite paths separated by cut times, the forward loop-erasure of the path $\langle S(n)\rangle_{n \leq 0}$ is well defined, which we denote by $\mathbf{L E}[S(-\infty, 0]]$. By the reversibility of the loop-erasing operation, the path $\mathbf{L E}[S(-\infty, 0]]$ has the same law as $\mathbf{L E}[S[0, \infty)]$. Now we use the event $A:=\{\mathbf{L E}[S(-\infty, 0]] \cap S[1, \infty)=\emptyset\}$, which plays the same role as the event

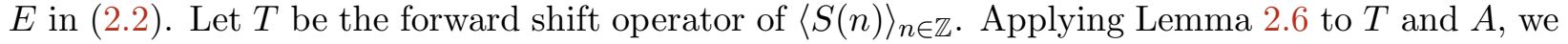
get Lemma 2.5.

Using estimates for random walk on $\mathbb{Z}^{d}$, it was shown in [Law80] that the two-sided LERW is weakly mixing, which is a property stronger than ergodicity. For this paper, we need only stationarity (see Section 4 for its use) and our argument can be readily extended to more general unimodular vertex-transitive graphs.

A network is a graph with positive weights on its edges. The corresponding network random walk is the Markov chain on its vertices that moves to a neighboring vertex of its present state with probabilities proportional to the weights of the incident edges. Constant weights correspond to simple random walk. For a vertex $x$ in a general transient network, $G$, define

$$
Z_{i}(x):=\sum_{t=0}^{\infty}(t+1)^{i} p_{t}(x, x) .
$$

When $G$ is $\mathbb{Z}^{d}$, or, more generally, transitive, $Z_{i}(x)$ does not depend on $x$, and so we will write simply $Z_{i}$ in the transitive case. Because $Z_{0}(x)$ is the expected number of visits to $x$ by a network random walk $S$ starting from $x$, we have that $Z_{0}(x)=1 / \mathbb{P}[\forall n \geq 1 S(n) \neq x]$. If $L(x)$ denotes the time of the last visit to $x$, it follows that for any loop $\gamma$ rooted at $x$, we have

$$
\mathbb{P}[S([0,|\gamma|])=\gamma] / Z_{0}(x)=\mathbb{P}[S([0, L(x)])=\gamma],
$$

and so

$$
\mathbb{E}[L(x)+1]=Z_{1}(x) / Z_{0}(x) .
$$

Lemma 2.7. Let $G$ be a network and $x$ be a vertex of $G$. Let $A$ be a set of vertices such that there is positive probability that the network walk $S$ from $x$ avoids $A$ forever: $\mathbb{P}[\forall n \geq 0 S(n) \notin A]>0$. Let $L:=\sup \{n \geq 0 ; S(n)=x\}$ be the last time the walk is at $x$. Then the probability that the first $L$ steps of the walk is equal to a particular loop $\gamma$ given that $A$ is avoided forever is at most the unconditional probability that the first $|\gamma|$ steps of the random walk is $\gamma$ :

$$
\mathbb{P}[S([0, L])=\gamma \mid \forall n \geq 0 \quad S(n) \notin A] \leq \mathbb{P}[S([0,|\gamma|])=\gamma] .
$$

Proof. This is a simple modification of the proof of the display after (5.4) of [Hut19]. To be more precise, given a path $\gamma$ avoiding $A$, we have

$$
\begin{aligned}
\mathbb{P}[S([0, L])=\gamma \mid \forall n \geq 0 \quad S(n) \notin A] & =\frac{\mathbb{P}[S([0,|\gamma|])=\gamma, \forall n>|\gamma| S(n) \notin A \cup\{x\}]}{\mathbb{P}[\forall n \geq 0 S(n) \notin A]} \\
& =\frac{\mathbb{P}[S([0,|\gamma|])=\gamma] \cdot \mathbb{P}[\forall n>|\gamma| S(n) \notin A \cup\{x\}]}{\mathbb{P}[\forall n \geq 0 S(n) \notin A]} \\
& \leq \mathbb{P}[S([0,|\gamma|])=\gamma] .
\end{aligned}
$$


Proposition 2.8. Let $G$ be a transitive network and o be a vertex of $G$. Consider the network walk $S$ from o. Define $K_{i}:=\#\{s ;|\mathbf{L E}[S([0, s])]|=i\}$. Then $\mathbb{E}\left[K_{i}\right] \leq Z_{1}$ for all $i \geq 0$.

Proof. That $\mathbb{E}\left[K_{0}\right]=Z_{0} \leq Z_{1}$ is trivial. Define $T_{0}:=0$ and for $i \in \mathbb{N}$, let

$$
T_{i}:=1+\sup \left\{t \geq T_{i-1} ; S(t)=S\left(T_{n-1}\right)\right\} .
$$

Then $\mid \mathbf{L E}\left(\left[S\left[0, T_{j}\right]\right) \mid=j\right.$ for all $j \geq 0$. Defining $K_{i, j}:=\#\left\{s \in\left[T_{j}, T_{j+1}\right) ;|\mathbf{L E}[S([0, s])]|=i\right\}$ for $0 \leq j \leq i$, we have $K_{i}=\sum_{j=0}^{i} K_{i, j}$.

Let $S^{\prime}$ be an independent simple random walk from $o$; let $T^{\prime}$ be one more than the last time $S^{\prime}$ visits $o$. Let $R_{j}:=\left\{s \in\left[0, T^{\prime}\right) ;\left|\mathbf{L E}\left[S^{\prime}([0, s])\right]\right|=i-j\right\}$; these sets are pairwise disjoint. By Lemma 2.7 and equation (2.3), for $i \geq j \geq 0$ and $k \geq 0$,

$$
\mathbb{P}\left[K_{i, j}=k \mid S\left[0, T_{j}\right]\right] \leq Z_{0} \mathbb{P}\left[\# R_{j}=k\right],
$$

whence

$$
\mathbb{E}\left[K_{i}\right]=\sum_{j=0}^{i} \mathbb{E}\left[K_{i, j}\right] \leq \sum_{j=0}^{i} Z_{0} \mathbb{E}\left[\# R_{j}\right]=Z_{0} \mathbb{E}\left[\# \bigcup_{j=0}^{i} R_{j}\right] \leq Z_{0} \mathbb{E}\left[T^{\prime}\right]=Z_{1},
$$

where, in the last step, we used (2.4).

\section{Recurrence when $d \geq 8$}

In this section, we first prove Theorem 1.1 and then extend the result to vertex-transitive graphs in Section 3.2. Recall $\mathcal{T}_{o}$ and $\mathrm{Ray}_{o}$ as in Definition 1.2 for the origin $o$ of $\mathbb{Z}^{d}$. Given $n \in \mathbb{N} \cup\{0\}$, we call the connected component of $\mathcal{T}_{o} \backslash\left(\operatorname{Ray}_{o}[0, n-1] \cup \operatorname{Ray}_{o}[n+1, \infty)\right)$ containing $\operatorname{Ray}_{o}(n)$ the $n$th $\boldsymbol{b u s h}$ of $\mathcal{T}_{o}$ and denote it by $\operatorname{Bush}_{n}$. Given an edge $e$ in $\mathbb{Z}^{d}$ and two subgraphs $H_{1}$ and $H_{2}$ of $\mathbb{Z}^{d}$ with $V\left(H_{1}\right) \cap V\left(H_{2}\right)=\varnothing$, we say that $e$ joins $H_{1}$ and $H_{2}$ if one endpoint of $e$ is in $H_{1}$ and the other is in $\mathrm{H}_{2}$.

Lemma 3.1. For $0 \leq j \leq n$ and $\ell \geq 1$, let $N_{j, \ell}(n)$ be the number of edges joining Bush $_{n-j}$ and Bush $_{n+\ell}$. Suppose $d \geq 8$. Then there exists a constant $C>0$ such that

$$
\sum_{0 \leq j \leq n} \sum_{\ell \geq m} \mathbb{E}\left[N_{j, \ell}(n)\right] \leq C \log \left(1+\frac{n}{m}\right) \quad \text { for all } m, n \in \mathbb{N} .
$$

We postpone the proof of Lemma 3.1 to Section 3.1 and proceed to prove Theorem 1.1.

Proof of Theorem 1.1. By Lemma 2.4, we see that Theorem 1.1 is equivalent to the statement that $\overline{\mathcal{T}_{o}}$ is recurrent a.s.

By (3.1) with $m=1$, there exists a constant $C>0$ and a sequence $n_{k} \in\left[k^{2 k}, 2 k^{2 k}\right]$ such that

$$
\sum_{0 \leq j \leq n_{k}} \sum_{\ell \geq 1} \mathbb{E}\left[N_{j, \ell}\left(n_{k}\right)\right] \leq C \log n_{k} \quad \text { for all } k \in \mathbb{N} .
$$

Define $\mathcal{C}_{k}$ to be the set consisting of edges joining $\bigcup_{m \leq n_{k}} \operatorname{Bush}_{m}$ and $\bigcup_{m>n_{k}} \operatorname{Bush}_{m}$. Then removing $\mathcal{C}_{k}$ from $\overline{\mathcal{T}_{o}}$ leaves $o$ in a finite component. Since $\mathbb{E}\left[\# \mathcal{C}_{k}\right] \lesssim \log n_{k}$ by $(3.2)$ and

$$
\sum_{k=1}^{\tau} \log \left(n_{k}\right) \lesssim \tau^{2} \log \tau \quad \text { for all } \tau \geq 1
$$


the argument in [BLPS01, Lemma 13.5 and Remark 13.6] yields $\sum_{1}^{\infty}\left(\# \mathcal{C}_{k}\right)^{-1}=\infty$ a.s.

Let $I_{k}$ be the event that there exists an edge joining $\bigcup_{m \leq n_{k}}$ Bush $_{m}$ and $\bigcup_{m \geq n_{k+1}} \mathrm{Bush}_{m}$. Since $\sum_{1}^{\infty} \frac{n_{k}}{n_{k+1}-n_{k}}<\infty$, by (3.1) and the Borel-Cantelli lemma, we know that almost surely only finitely many events $I_{k}$ occur. Therefore there exists a (random) $K \in \mathbb{N}$ such that the elements in $\left\{\mathcal{C}_{k} ; k \geq\right.$ $K\}$ are all disjoint. By the Nash-Williams criterion (see, e.g. [LP16, Sec. 2.5]), it follows that $\overline{\mathcal{T}_{o}}$ is recurrent a.s.

\subsection{Proof of Lemma 3.1}

Fix $d \in \mathbb{N}$. For $z \in \mathbb{Z}^{d}$, let $\Omega^{z}$ be the space of loops in $\mathbb{Z}^{d}$ rooted at $z$ (see Section 2.1 for the definition). Define a measure $\mu$ on $\Omega^{z}$ by requiring $\mu(\gamma):=(2 d)^{-|\gamma|}$ for all $\gamma \in \Omega^{z}$. We call $\mu$ the loop measure and $\mu(\gamma)$ the weight of $\gamma$. Here we drop the dependence of $\mu$ on $z$ for simplicity of notation. In different places, we will consider loops with additional markings. For example, let $\Omega_{\mathrm{s}}^{z}:=\left\{(\gamma, i) ; \gamma \in \Omega^{z}, i \in[0,|\gamma|-1] \cap \mathbb{Z}\right\}$. Each element in $\Omega_{\mathrm{s}}^{z}$ is a loop $\gamma$ rooted at $z$ with a marked step being the ordered pair $\langle\gamma(i), \gamma(i+1)\rangle$. By assigning each element in $\Omega_{\mathrm{s}}^{z}$ the weight of its loop, we define a measure on $\Omega_{\mathrm{s}}^{z}$, which we still denote by $\mu$ in a slight abuse of notation.

Proof of Lemma 3.1. By linearity of expectation, we estimate $\mathbb{E}\left[N_{j, \ell}(n)\right]$ by estimating the probability of joining Bush $_{n-j}$ and Bush $_{n+\ell}$ for each edge of $\mathbb{Z}^{d}$. Let $x$ and $y$ be two adjacent vertices in $\mathbb{Z}^{d}$ and $S, S^{1}$, and $S^{2}$ be three independent simple random walks on $\mathbb{Z}^{d}$ starting from $o, x$, and $y$ respectively. Suppose that $\mathrm{WSF}\left(\mathbb{Z}^{d}\right)$ is sampled via Wilson's algorithm rooted at infinity by first sampling $S, S^{1}$, and $S^{2}$, and then other random walks. Fix $0 \leq j \leq n$ and $\ell, m \in \mathbb{N}$. Given $s, s^{\prime}, t^{\prime} \in \mathbb{N} \cup\{0\}$ and $z, w \in \mathbb{Z}^{d}$, let $E^{x, y}\left(s, s^{\prime}, t^{\prime}, z, w\right)$ be the event that

1. $S(s)=z$ and $|\mathbf{L E}[S([0, s])]|=n-j$;

2. $S^{1}\left(s^{\prime}\right)=z$ and $S^{2}\left(t^{\prime}\right)=w$;

3. $\lambda:=\sup \{k ; S(k)=w\} \in[s, \infty)$ and $|\mathbf{L E}[S([s, \lambda])]|=j+\ell$; and

4. $t^{\prime}=\inf \left\{k ; S^{2}(k) \in \mathbf{L E}[S([s, \lambda])]\right\}$.

Then $\left\{x \in V\left(\operatorname{Bush}_{n-j}\right)\right.$ and $\left.y \in V\left(\operatorname{Bush}_{n+\ell}\right)\right\} \subset \bigcup E^{x, y}\left(s, s^{\prime}, t^{\prime}, z, w\right)$, where the union ranges over all possible tuples $\left(s, s^{\prime}, t^{\prime}, z, w\right)$.

Recall $\Omega_{\mathrm{s}}^{z}$ defined right above. Let $\Omega_{\mathrm{s}}^{z}\left(x, y, w, s^{\prime}, t^{\prime}\right) \subset \Omega_{\mathrm{s}}^{z}$ be the set of $(\gamma, i)$ that satisfy

1. $\gamma(i)=y$ and $\gamma(i+1)=x$;

2. $s^{\prime}=|\gamma|-i-1$;

3. $\mathbf{L E}[\gamma([0, i])](j+\ell)=w$; and

4. $\max \{k \leq i ; \gamma(k)=w\}=i-t^{\prime}$.

On $E^{x, y}\left(s, s^{\prime}, t^{\prime}, z, w\right)$, by concatenating $S([s, \lambda])$, the reversal of $S^{2}\left(\left[0, t^{\prime}\right]\right)$, the edge from $y$ to $x$, and $S^{1}\left(\left[0, s^{\prime}\right]\right)$, we obtain an element in $\Omega_{\mathrm{s}}^{z}\left(x, y, w, s^{\prime}, t^{\prime}\right)$ whose marked step is $\langle y, x\rangle$. Therefore $\mathbb{P}\left[E^{x, y}\left(s, s^{\prime}, t^{\prime}, z, w\right)\right]$ equals

$$
\mathbb{P}[S(s)=z \text { and }|\mathbf{L E}[S([0, s])]|=n-j] \cdot(2 d) \cdot \mu\left[\Omega_{\mathrm{s}}^{z}\left(x, y, w, s^{\prime}, t^{\prime}\right)\right],
$$

where the factor $2 d$ comes from the fact that the step $\langle y, x\rangle$ need not be traversed by $S, S^{1}$, or $S^{2}$. Note that $\Omega_{\mathrm{s}}^{z}\left(x, y, w, s^{\prime}, t^{\prime}\right) \subset\left\{(\gamma, i) \in \Omega_{\mathrm{s}}^{z} ;|\gamma| \geq j+\ell\right\}$. Now let $x$ and $y$ vary. For different tuples 
$\left(x, y, w, s^{\prime}, t^{\prime}\right)$, the corresponding sets $\Omega_{\mathrm{s}}^{z}\left(x, y, w, s^{\prime}, t^{\prime}\right)$ are disjoint (because the definition (1)-(4) determines $x, y, w, s^{\prime}$ and $t^{\prime}$ from $\left.(\gamma, i)\right)$. Therefore

$$
\sum_{x, y, w, s^{\prime}, t^{\prime}} \mu\left[\Omega_{\mathrm{s}}^{z}\left(x, y, w, s^{\prime}, t^{\prime}\right)\right] \leq \mu\left[\left\{(\gamma, i) \in \Omega_{\mathrm{s}}^{z} ;|\gamma| \geq j+\ell\right\}\right] .
$$

Let $p$ be the transition kernel of $\mathbb{Z}^{d}$. By the definition of $\Omega_{\mathrm{s}}^{z}$ and $\mu$, for all $t \in \mathbb{N}$, we have

$$
\mu\left[\left\{(\gamma, i) \in \Omega_{\mathrm{s}}^{z} ;|\gamma|=t\right\}\right]=t p_{t}(z, z)=t p_{t}(o, o) .
$$

Since $d \geq 8$ and $p_{t}(o, o) \lesssim t^{-d / 2}$, we see from (3.4) that

$$
\sum_{x, y, w, s^{\prime}, t^{\prime}} \mu\left[\Omega_{\mathrm{s}}^{z}\left(x, y, w, s^{\prime}, t^{\prime}\right)\right] \leq \sum_{t \geq j+\ell} t p_{t}(o, o) \lesssim(j+\ell)^{-2} .
$$

Set $K_{i}:=\#\{s ;|\mathbf{L E}[S([0, s])]|=i\}$ for all $0 \leq i \leq n$, as in Proposition 2.8. By (3.3), we have

$$
\begin{aligned}
& \sum_{x, y, s, s^{\prime} t^{\prime}, z, w} \mathbb{P}\left[E^{x, y}\left(s, s^{\prime}, t^{\prime}, z, w\right)\right] \lesssim(j+\ell)^{-2} \sum_{s, z} \mathbb{P}[S(s)=z,|\mathbf{L E}[S([0, s])]|=n-j] \\
&=(j+\ell)^{-2} \sum_{s} \mathbb{P}[|\mathbf{L E}[S([0, s])]|=n-j]=\mathbb{E}\left[K_{n-j}\right](j+\ell)^{-2} \lesssim(j+\ell)^{-2}
\end{aligned}
$$

by Proposition 2.8. Since $\mathbb{E}\left[N_{j, \ell}(n)\right] \leq \sum_{x, y, s, s^{\prime} t^{\prime}, z, w} \mathbb{P}\left[E^{x, y}\left(s, s^{\prime}, t^{\prime}, z, w\right)\right]$, we see that

$$
\sum_{0 \leq j \leq n} \sum_{\ell \geq m} \mathbb{E}\left[N_{j, \ell}(n)\right] \lesssim \sum_{0 \leq j \leq n} \sum_{\ell \geq m}(j+\ell)^{-2} \lesssim \sum_{0 \leq j \leq n}(j+m)^{-1} \lesssim \log (1+n / m)
$$

\subsection{Extension to vertex-transitive graphs}

Suppose $G$ is a vertex-transitive graph and $o \in V(G)$. We call $V(r):=\# B_{G}(o, r)$ the volume growth function of $G$. In this subsection, we explain the following extension of Theorem 1.1.

Theorem 3.2. If $G$ is a vertex-transitive graph with $V(r) \gtrsim r^{8}$, then almost surely each connected component of $\overline{\mathrm{WSF}(G)}$ is recurrent.

Let $p$ be the transition kernel of $G$. It is standard that $p_{t}(o, o) \lesssim t^{-4}$ when $V(r) \gtrsim r^{8}$ (see, e.g., [LP16, Corollary 6.32]). Given this, Proposition 2.8 implies Lemma 3.1, hence Theorem 1.1 in the same way with $\mathbb{Z}^{d}$ replaced by $G$ in Theorem 1.1 .

If $G$ is nonunimodular, then Theorem 3.2 is essentially already known (see the definition of unimodular preceding Theorem 4.3). In fact, it is well known that $G$ is nonamenable in this case, that is, $\inf _{K} \# \partial K / \# K>0$, where the infimum is over all finite vertex sets $K$ of $G$; see, e.g., [LP16, Proposition 8.14]. Therefore, by [BLPS01, Theorem 13.1], we have $\mathbb{E}\left[\#\left(\overline{\mathcal{T}_{o}} \cap B_{G}(0, n)\right)\right] \asymp n^{2}$, where $\mathcal{T}_{o}$ is the component of $\operatorname{WSF}(G)$ containing $o$. Now [BLPS01, Lemma 13.5] yields that $\overline{\mathcal{T}_{o}}$ is a.s. recurrent. Since $G$ is transient, Lemma 2.4 concludes Theorem 3.2 in the nonunimodular case.

\section{Linear growth of resistance when $d \geq 9$}

Recall the two-sided LERW defined in Section 2.5. Now we define the two-sided WSF.

Definition 4.1. Given $d \geq 5$, sample a random spanning forest $\mathfrak{F}_{\mathrm{w}}^{2}\left(\mathbb{Z}^{d}\right)$ on $\mathbb{Z}^{d}$ as follows. 
1. Sample a two-sided loop-erased random walk $\widetilde{S}$.

2. Conditioning on $\widetilde{S}$, sample a WSF (denoted by $\mathfrak{F}_{\mathrm{w}}$ ) on the graph obtained from $\mathbb{Z}^{d}$ by identifying the trace of $\widetilde{S}$ as a single vertex.

3. Set $\mathfrak{F}_{\mathrm{w}}^{2}\left(\mathbb{Z}^{d}\right)$ to be the union of $\mathfrak{F}_{\mathrm{w}}$ and the trace of $\widetilde{S}$, where $\mathfrak{F}_{\mathrm{w}}$ is viewed as a random subgraph of $\mathbb{Z}^{d}$.

We call the law of $\mathfrak{F}_{\mathrm{w}}^{2}\left(\mathbb{Z}^{d}\right)$ the two-sided wired spanning forest on $\mathbb{Z}^{d}$ and denote it by $\mathrm{WSF}^{2}\left(\mathbb{Z}^{d}\right)$.

It is clear that $\mathrm{WSF}^{2}\left(\mathbb{Z}^{d}\right)$ can be sampled from a modified version of Wilson's algorithm rooted at infinity: first sample a two-sided LERW and treat it as the first walk in Wilson's algorithm; then proceed as in the original Wilson's algorithm to form a spanning forest on $\mathbb{Z}^{d}$. The stationary two-sided LERW on $\mathbb{Z}^{d}$ was extended to $d=4$ in [LSW19] and to $d=2,3$ in [Law18] by a limiting procedure. Therefore $\operatorname{WSF}^{2}\left(\mathbb{Z}^{d}\right)$ can be defined for all $d \in \mathbb{N}$. However, we will not need the lower-dimensional cases.

By Lemma 2.5, as a subgraph-decorated rooted graph, $\left(\mathbb{Z}^{d}, o, \mathfrak{F}_{\mathrm{w}}^{2}\left(\mathbb{Z}^{d}\right)\right)$ is stationary under shifting along the trace of $\widetilde{S}$. We will use this stationarity and the ergodic theorem to prove Theorem 1.3. The following lemma will be needed.

Lemma 4.2. In the setting of Theorem 1.3, for $v \in \mathbb{Z}^{d}$ such that $v \neq o$, let $\mathcal{N}_{v}$ be the number of edges joining $\mathcal{T}_{o}$ and $\mathcal{T}_{v}$ if $\mathcal{T}_{o} \neq \mathcal{T}_{v}$ and be 0 otherwise. Then $\mathbb{E}\left[\mathcal{N}_{v}\right]<\infty$ for $d \geq 9$.

Proof. We follow an argument similar to that in Lemma 3.1. Given two neighboring vertices $x$ and $y$, let $\mathcal{I}_{x, y}$ be the event that $x \in \mathcal{T}_{o}$ and $y \in \mathcal{T}_{v}$. Suppose that $S^{o}, S^{v}, S^{x}$, and $S^{y}$ are independent simple random walks on $\mathbb{Z}^{d}$ starting from $o, v, x$, and $y$, respectively. By Wilson's algorithm,

$$
\mathbb{P}\left[\mathcal{I}_{x, y}\right] \leq \mathbb{P}\left[S^{x}([0, \infty)) \cap S^{o}([0, \infty)) \neq \varnothing \text { and } S^{y}([0, \infty)) \cap S^{v}([0, \infty)) \neq \varnothing\right] .
$$

Therefore

$$
\mathbb{E}\left[\mathcal{N}_{v}\right] \leq \sum_{x \sim y} \sum_{k, l, m, n \geq 0} \mathbb{P}\left[S^{o}(k)=S^{x}(l) \text { and } S^{y}(m)=S^{v}(n)\right]
$$

Now let $\Omega^{o, v}$ be the space of quadruples $(\gamma, \sigma, \tau, i)$ where $\gamma$ is a path in $\mathbb{Z}^{d}$ from $o$ to $v, \sigma, \tau \in$ $[0,|\gamma|] \cap \mathbb{Z}$, and $i \in[0,|\gamma|-1] \cap \mathbb{Z}$. Here, $\sigma$ and $\tau$ are considered as two marked times of $\gamma$ and $\langle\gamma(i), \gamma(i+1)\rangle$ is considered as a marked step. Define the measure $\mu$ on $\Omega^{o, v}$ by assigning weight $(2 d)^{-|\gamma|}$ to each $(\gamma, \sigma, \tau, i) \in \Omega^{o, v}$. Then

$$
\mu\left[\Omega^{o, v}\right]=\sum_{t=0}^{\infty} \mu\left[\left\{(\gamma, \sigma, \tau, i) \in \Omega^{o, v} ;|\gamma|=t\right\}\right]=\sum_{t=0}^{\infty} t(t+1)^{2} p_{t}(o, v) .
$$

Since $p_{t}(o, v) \lesssim t^{-d / 2}$ (see [LP16, Corollary 6.32(ii)]), we see that $\mu\left[\Omega^{o, v}\right]<\infty$ if $d \geq 9$.

Let $\Omega^{o, v}(k, l, m, n):=\left\{(\gamma, k, k+\ell+1+m, k+\ell) \in \Omega^{o, v} ;|\gamma|=k+\ell+1+m+n\right\}$. By concatenating $S^{o}([0, k])$, the reversal of $S^{x}([0, \ell])$, the edge from $x$ to $y$, the path $S^{y}([0, m])$, and the reversal of $S^{v}([0, n])$, we see that $\sum_{x \sim y} \mathbb{P}\left[S^{o}(k)=S^{x}(l)\right.$ and $\left.S^{y}(m)=S^{v}(n)\right]$ is no larger than $\mu\left[\Omega^{o, v}(k, l, m, n)\right]$. On the other hand, $\Omega^{o, v}(k, l, m, n) \cap \Omega^{o, v}\left(k^{\prime}, l^{\prime}, m^{\prime}, n^{\prime}\right)=\varnothing$ if $(k, l, m, n) \neq$ $\left(k^{\prime}, l^{\prime}, m^{\prime}, n^{\prime}\right)$. Now interchanging the summations in (4.1), we get $\mathbb{E}\left[\mathcal{N}_{v}\right] \leq \mu\left[\Omega^{o, v}\right]$, which is finite if $d \geq 9$.

\footnotetext{
${ }^{2}$ Recall the notion from Section 3.
} 
Proof of Theorem 1.3. By Lemma 2.4, it suffices to prove that

$$
\mathbb{P}\left[\liminf _{n \rightarrow \infty} n^{-1} \mathrm{R}_{\text {eff }}^{\overline{\mathcal{T}_{o}}}\left(o, \operatorname{Ray}_{o}(n)\right)>0\right]>0 .
$$

Let us make a particular choice in Wilson's algorithm rooted at infinity to sample WSF $(G)$.

1. Sample a simple random walk $S^{1}$ from $o$ as the first walk in Wilson's algorithm.

2. Run an independent simple random walk $S^{2}$ from $o$. Define $\tau:=\inf \left\{t \geq 1 ; S^{2}(t) \notin \mathbf{L E}\left[S^{1}\right]\right\}$ and $v:=S^{2}(\tau)$.

3. Use $S^{2}([\tau, \infty))$ for the second simple random walk in Wilson's algorithm.

4. Sample the rest of $\operatorname{WSF}(G)$ according to Wilson's algorithm in an arbitrary way.

Let $\mathbb{P}$ be the probability measure from the above sampling and let $\widetilde{\mathbb{P}}$ be $\mathbb{P}$ conditioned on the event $B:=\left\{\tau=1\right.$ and $\left.\mathcal{T}_{v} \neq \mathcal{T}_{o}\right\}$. Then $B$ is exactly the event $E$ in (2.2). We define $\widetilde{S}$ in terms of $\left(S^{1}, S^{2}\right)$ as in Lemma 2.5, so that under $\widetilde{\mathbb{P}}$ it is a two-sided LERW. On the event $B$, let $\mathfrak{F}_{\mathrm{w}}^{2}$ consist of the edges of $\operatorname{WSF}(G)$ and the edge $(o, v)$, and let $\widetilde{\mathcal{T}}_{o}$ consist of the edges of $\mathcal{T}_{o}$ and $\mathcal{T}_{v}$ and the edge $(o, v)$. By Lemma 2.5 and Definition 4.1, under $\widetilde{\mathbb{P}}$, we see that $\mathfrak{F}_{\mathrm{w}}^{2}$ is distributed as $\operatorname{WSF}^{2}(G)$ and $\widetilde{\mathcal{T}}_{o}$ is the component of $\mathfrak{F}_{\mathrm{w}}^{2}$ containing $o$.

To prove (4.2), recall the notion of $\mathrm{Bush}_{n}$ in Section 3. For $k \in \mathbb{N}$, let $\mathcal{C}_{k}$ be the set of edges joining $\bigcup_{m \leq k} \operatorname{Bush}_{m}$ and $\bigcup_{m \geq k+1} \operatorname{Bush}_{m}$. For any edge $e$ of $\mathbb{Z}^{d}$, let $j(e):=\#\left\{k ; e \in \mathcal{C}_{k}\right\}$. Let $J_{k}:=\sum_{e \in \mathcal{C}_{k}} j(e)$. Under $\widetilde{\mathbb{P}}$, for $n \in \mathbb{Z}$, let $\widetilde{\operatorname{Bush}_{n}}$ be the connected component of $\widetilde{\mathcal{T}_{o}} \backslash \widetilde{S}(\mathbb{Z} \backslash\{n\})$ containing $\widetilde{S}(n)$. Let $\widetilde{\mathcal{C}_{k}}$ be the set of edges joining $\bigcup_{m \leq k} \widetilde{\operatorname{Bush}}_{m}$ and $\bigcup_{m \geq k+1} \widetilde{\operatorname{Bush}}_{m}$. Let $\widetilde{j}(e):=$ $\#\left\{k ; e \in \widetilde{\mathcal{C}}_{k}\right\}$ and $\widetilde{J}_{k}:=\sum_{e \in \widetilde{\mathcal{C}}_{k}} \widetilde{j}(e)$. By Lemma $4.2, \# \widetilde{\mathcal{C}_{-1}}<\infty \widetilde{\mathbb{P}}$-a.s. By the stationarity of $\operatorname{WSF}^{2}\left(\mathbb{Z}^{d}\right)$, both $\left\langle\widetilde{\mathcal{C}}_{k}\right\rangle_{k \in \mathbb{Z}}$ and $\left\langle\widetilde{J}_{k}\right\rangle_{k \in \mathbb{Z}}$ are stationary under $\widetilde{\mathbb{P}}$. On the other hand, if $e \in \mathcal{C}_{k}$ joins $\operatorname{Bush}_{m}$ and $\operatorname{Bush}_{n}$ for some $n>m$, we must have $e \in \widetilde{\mathcal{C}}_{k}$ and $j(e)=\widetilde{j}(e)=n-m$. Therefore $\mathcal{C}_{k} \subset \widetilde{\mathcal{C}_{k}}$ and $J_{k} \leq \widetilde{J}_{k}<\infty \widetilde{\mathbb{P}}$-a.s. for all $k \in \mathbb{N} \cup\{0\}$. By the stationarity of $\left\langle\widetilde{J}_{k}\right\rangle_{k \in \mathbb{Z}}$ under $\widetilde{\mathbb{P}}$ and Birkhoff's ergodic theorem, there exists a random variable $Y$ such that $\mathbb{E}^{\widetilde{\mathbb{P}}}[Y]=\mathbb{E}^{\widetilde{\mathbb{P}}}\left[\widetilde{J}_{0}^{-1}\right]>0$ and $\lim _{n \rightarrow \infty} n^{-1} \sum_{k=0}^{n-1} \widetilde{J}_{k}^{-1}=Y \widetilde{\mathbb{P}}$-a.s. Since $J_{k} \leq \widetilde{J}_{k}$, with positive probability under $\widetilde{\mathbb{P}}$ (hence under $\mathbb{P})$, we have

$$
\liminf _{n \rightarrow \infty} n^{-1} \sum_{k=0}^{n-1} J_{k}^{-1}>0 .
$$

By Lemma 2.1, $\mathrm{R}_{\text {eff }}^{\overline{T_{o}}}\left(o, \operatorname{Ray}_{o}(n)\right) \geq \sum_{k=0}^{n-1} J_{k}^{-1}$, which gives (4.2).

We conclude this section by the following straightforward extension of Theorem 1.3. Let $S$ be the two-sided random walk on $G$ defined as in Section 2.5 with $\mathbb{Z}^{d}$ and its origin replaced by $G$ and $o \in V(G)$. A vertex-transitive graph $G$ is unimodular if the automorphism group $\operatorname{Aut}(G)$ of $G$ is unimodular, in other words, $\operatorname{Aut}(G)$ admits a nontrivial Borel measure that is invariant under both left and right multiplication by group elements. In this case, $G$ satisfies $^{3}$

$$
\langle S(n)\rangle_{n \in \mathbb{Z}} \text { is stationary and ergodic viewed as path-decorated rooted graphs. }
$$

We will not elaborate on the notion of unimodularity, but refer to [BLPS99] or [LP16, Chapter 8] for more background.

\footnotetext{
${ }^{3}$ It can be shown that unimodularity is, in fact, equivalent to (4.3).
} 
Theorem 4.3. Theorem 1.3 still holds if $\mathbb{Z}^{d}$ is replaced by a unimodular, vertex-transitive graph $G$ such that $V(r) \gtrsim r^{9}$. (Recall the function $V(r)$ in Section 3.2.)

Proof. Note that $V(r) \gtrsim r^{9}$ implies that the transition kernel satisfies $p_{t}(o, o) \lesssim t^{-9 / 2}$. By inspection, the proof of Theorem 1.3 still works given this transition-kernel estimate and the fact that $\mathcal{T}_{o}$ can be coupled with the (stationary) two-sided WSF as in the proof of Theorem 1.3 via Wilson's algorithm. This holds as long as the two-sided LERW can be sampled from the two-sided simple random walk as in Section 2.5. By (4.3) and Lemma 2.6, this is true if $G$ is unimodular.

We expect that the unimodularity assumption in Theorem 4.3 can be removed. However, this would require a different approach, because for nonunimodular, vertex-transitive graphs, although the two-sided LERW can still be defined by a limiting procedure, it is not related to the two-sided simple random walk that we defined earlier.

\section{$5 \quad$ Resampling property}

In this section, we first prove Theorem 1.5 and Corollary 1.7 in Section 5.1. Then we provide a counterexample to $\operatorname{WSF}\left(\overline{\mathfrak{F}_{\mathrm{f}}(G)}\right)=\operatorname{WSF}(G)$ in Section 5.2.

\subsection{Proof of Theorem 1.5 and Corollary 1.7}

We introduce the following notation. Given a graph $G$, suppose $H$ is a random finite subgraph of $G$. Let us sample a random forest on $G$ as follows. First sample $H$. Conditioning on $H$, uniformly sample a spanning tree on each component of $H$. The unconditional law of the resulting random forest is denoted by $\operatorname{USF}(H)$.

Proof of Theorem 1.5. We prove only FSF $\left(\overline{\mathfrak{F}_{\mathrm{W}}(G)}\right)=\operatorname{WSF}(G)$ since $\operatorname{FSF}\left(\overline{\mathfrak{F}_{\mathrm{f}}(G)}\right)=\operatorname{FSF}(G)$ can be proved in exactly the same way.

Fix $o \in V(G)$. For a positive integer $n$, let $\mathfrak{F}_{\mathrm{w}}^{n}$ be a sample of $\operatorname{WSF}\left(B_{G}(o, n)\right)$. For $0<m<n$, thinking of $\mathfrak{F}_{\mathrm{w}}^{n} \cap B_{G}(o, m)$ and $\mathfrak{F}_{\mathrm{w}} \cap B_{G}(o, m)$ as subgraphs of $B_{G}(o, m)$, let $K_{m, n}$ and $K_{m}$ be their induced-component graphs, respectively. For a fixed $m$, as $n$ tends to $\infty$, the laws of $\mathfrak{F}_{\mathrm{w}}^{n} \cap B_{G}(o, m)$ and $\mathfrak{F}_{\mathrm{w}} \cap B_{G}(o, m)$ can be coupled so that they are identical with probability $1-o_{n}(1)$. Hence the same is true for $K_{m, n}$ and $K_{m}$. Conditioning on $K_{m, n}=K$, the conditional law of $\mathfrak{F}_{\mathrm{w}}^{n} \cap B_{G}(o, m)$ is $\operatorname{USF}(K)$ because every spanning forest of $K$ that is connected in each component of $K$ extends to a spanning tree of $\widehat{B_{G}(o, n)}$ in the same number of ways. Letting $n$ tend to $\infty$, we see that the law of $\mathfrak{F}_{\mathrm{w}} \cap B_{G}(o, m)$ is $\operatorname{USF}\left(K_{m}\right)$. Note that $\left\langle K_{m}\right\rangle_{m \geq 1}$ is an exhaustion of $\overline{\mathfrak{F}_{\mathrm{w}}(G)}$. (More precisely, each component of $\overline{\mathfrak{F}_{\mathrm{W}}(G)}$ is exhausted by the corresponding sequence of components of $K_{m}$.) Therefore by the definition of FSF, the measures $\operatorname{USF}\left(K_{m}\right)$ converge to $\operatorname{FSF}\left(\overline{\mathfrak{F}_{\mathrm{w}}(G)}\right)$ as $m \rightarrow \infty$ (restricted to any finite subgraph of $G$ ). Since the law of $\mathfrak{F}_{\mathrm{w}} \cap B_{G}(o, m)$ is $\operatorname{USF}\left(K_{m}\right)$, by letting $m$ tend to $\infty$, we obtain $\operatorname{WSF}(G)=\operatorname{FSF}\left(\overline{\mathfrak{F}_{\mathrm{w}}(G)}\right)$.

Proof of Corollary 1.7. Recall that $\operatorname{WSF}(G) \preccurlyeq \operatorname{FSF}(G)$ for any locally finite connected graph $G$ (see, e.g., [LP16, Section 10.2]). Together with Theorem 1.5, we obtain

$$
\operatorname{WSF}\left(\overline{\mathfrak{F}_{\mathrm{w}}(G)}\right) \preccurlyeq \operatorname{FSF}\left(\overline{\mathfrak{F}_{\mathrm{w}}(G)}\right)=\operatorname{WSF}(G) .
$$

Let $\left(\mathfrak{F}_{\mathrm{w}}^{\prime}, \mathfrak{F}_{\mathrm{w}}\right)$ be a coupling of $\operatorname{WSF}\left(\overline{\mathfrak{F}_{\mathrm{w}}(G)}\right)$ and $\operatorname{WSF}(G)$ such that $\mathfrak{F}_{\mathrm{w}}^{\prime} \subset \mathfrak{F}_{\mathrm{w}}$. Since each connected component of $\mathfrak{F}_{\mathrm{w}}^{\prime}$ is an infinite graph a.s., while each component of $\mathfrak{F}_{\mathrm{w}}$ has a single end, we must have $\mathfrak{F}_{\mathrm{w}}=\mathfrak{F}_{\mathrm{w}}^{\prime}$ a.s. This proves the first assertion; the second is even simpler, because by Theorem 1.5, we need only show that $\operatorname{WSF}(G)=\operatorname{FSF}(G)$. 


\subsection{A counterexample for $\operatorname{WSF}(\overline{\operatorname{FSF}(G)})=\operatorname{WSF}(G)$}

Recall that for any graph $G$ and neighbors $x, y$ in $G$, Kirchhoff's formula extended to the wired spanning forest gives that $\mathbb{P}\left[(x, y) \in \mathfrak{F}_{\mathrm{w}}(G)\right]=\mathrm{R}_{\mathrm{w}-\mathrm{eff}}^{G}(x, y)$; see [LP16, Equation (10.3)]. Here, the wired effective resistance between $x$ and $y$ is defined by

$$
\mathrm{R}_{\mathrm{w}-\mathrm{eff}}^{G}(x, y)=\left(\inf \left\{\mathcal{E}(f) ; f(x)=1, f(y)=0, \#\left(f^{-1}[\mathbb{R} \backslash\{0\}]\right)<\infty\right\}\right)^{-1} .
$$

If $H$ is a subgraph of $G$ that includes $(x, y)$ but does not include at least one edge $(u, w)$ for which the wired current $i_{\mathrm{w}}^{(x, y)}(u, w) \neq 0$, then Thomson's principle (uniqueness of the minimizing-energy unit flow) yields $\mathbb{P}\left[(x, y) \in \mathfrak{F}_{\mathrm{w}}(G)\right]<\mathbb{P}\left[(x, y) \in \mathfrak{F}_{\mathrm{w}}(H)\right]$; see [LP16, Section 9.1] for the definition of wired current.

Now let $G$ be the graph consisting of two copies of $\mathbb{Z}^{5}$, which we denote by $\mathbb{Z}^{5} \times\{0\}$ and $\mathbb{Z}^{5} \times\{1\}$, and an edge $e$ connecting $o_{0}:=(o, 0)$ and $o_{1}:=(o, 1)$. As before, $o$ represents the origin of $\mathbb{Z}^{5}$. Since $\mathbb{Z}^{5}$ is transient, the wired current $i_{\mathrm{w}}^{e}$ is nonzero on infinitely many edges of $\mathbb{Z}^{5} \times\{i\}$ for each $i \in\{0,1\}$. (In fact, it can be proved that all edges have nonzero current.) Recall that $\operatorname{FSF}\left(\mathbb{Z}^{5}\right)=\operatorname{WSF}\left(\mathbb{Z}^{5}\right)$. Since $\mathfrak{F}_{\mathrm{f}}\left(\mathbb{Z}^{5}\right)$ contains infinitely many trees a.s., its induced components are not all of $\mathbb{Z}^{5}$. Furthermore, $e$ is not contained in any cycle, whence $e \in \mathfrak{F}_{\mathrm{f}}(G)$ a.s., and $\mathrm{FSF}(G)$ may be coupled with $\operatorname{FSF}\left(\mathbb{Z}^{5} \times\{0\}\right)$ and $\operatorname{FSF}\left(\mathbb{Z}^{5} \times\{1\}\right)$ so that $\mathfrak{F}_{\mathrm{f}}(G)=\{e\} \cup \mathfrak{F}_{\mathrm{f}}\left(\mathbb{Z}^{5} \times\{0\}\right) \cup \mathfrak{F}_{\mathrm{f}}\left(\mathbb{Z}^{5} \times\{1\}\right)$. Let $\mathcal{T}_{e}$ be the component of $\mathfrak{F}_{\mathrm{f}}(G)$ containing $e$. Then $\mathcal{T}_{e}$ consists of $e$ and the component of $\mathfrak{F}_{\mathrm{f}}\left(\mathbb{Z}^{5} \times\{i\}\right)$ containing $o_{i}$, where $i=0,1$. Each edge of $\mathbb{Z}^{5}$ has the same probability of being in $\overline{\mathfrak{F}_{\mathrm{f}}\left(\mathbb{Z}^{5}\right)}$, whence infinitely many edges $(u, w)$ with $i_{\mathrm{w}}^{e}(u, w) \neq 0$ are not in $\overline{\mathcal{T}_{e}}$ a.s. It follows from the preceding paragraph that $\mathbb{P}\left[(x, y) \in \mathfrak{F}_{\mathrm{w}}(G)\right]<\mathbb{P}\left[(x, y) \in \mathfrak{F}_{\mathrm{w}}\left(\overline{\mathcal{T}_{e}}\right) \mid \overline{\mathcal{T}_{e}}\right]$. Taking the expectation gives the result.

This same method answers negatively a long-standing question of whether $\operatorname{WSF}\left(\mathfrak{F}_{\mathrm{f}}(G)\right)=$ WSF $(G)$ for Cayley graphs, $G$. We may take $G$ to be the natural Cayley graph of the free product of $\mathbb{Z}^{3}$ with $\mathbb{Z}_{2}$ to obtain a counterexample. The analysis is similar to the preceding.

\section{References}

[BKPS04] I. Benjamini, H. Kesten, Y. Peres, and O. Schramm. Geometry of the uniform spanning forest: transitions in dimensions 4,8,12,... Ann. of Math. (2), 160(2):465-491, 2004. MR2123930 (2005k:60026)

[BLPS99] I. Benjamini, R. Lyons, Y. Peres, and O. Schramm. Group-invariant percolation on graphs. Geom. Funct. Anal., 9(1):29-66, 1999. MR99m:60149

[BLPS01] I. Benjamini, R. Lyons, Y. Peres, and O. Schramm. Uniform spanning forests. Ann. Probab., 29(1):1-65, 2001. MR1825141 (2003a:60015)

[BLS99] I. Benjamini, R. Lyons, and O. Schramm. Percolation perturbations in potential theory and random walks. In M. Picardello and W. Woess, editors, Random Walks and Discrete Potential Theory, Sympos. Math., pages 56-84, Cambridge, 1999. Cambridge University Press. Papers from the workshop held in Cortona, 1997. MR1802426

[Dub09] J. Dubédat. SLE and the free field: partition functions and couplings. J. Amer. Math. Soc., 22(4):995-1054, 2009. MR2525778

[HN17] T. Hutchcroft and A. Nachmias. Indistinguishability of trees in uniform spanning forests. Probab. Theory Related Fields, 168(1-2):113-152, 2017. MR3651050 
[HP19] T. Hutchcroft and Y. Peres. The component graph of the uniform spanning forest: transitions in dimensions 9, 10,11,... Probab. Theory Related Fields, 175(1-2):141-208, 2019. MR4009707

[Hut18] T. Hutchcroft. Interlacements and the wired uniform spanning forest. Ann. Probab., 46(2):1170-1200, 2018. MR3773383

[Hut19] T. Hutchcroft. Universality of high-dimensional spanning forests and sandpiles. Probab. Theory Related Fields, Jun 2019. http://dx.doi.org/10.1007/s00440-019-00923-3.

[Law80] G. F. Lawler. A self-avoiding random walk. Duke Math. J., 47(3):655-693, 1980. MR587173

[Law18] G. F. Lawler. The infinite two-sided loop-erased random walk. ArXiv e-prints, February 2018, 1802.06667.

[LMS08] R. Lyons, B. J. Morris, and O. Schramm. Ends in uniform spanning forests. Electron. J. Probab., 13:paper no. 58, 1702-1725, 2008. MR2448128

[LP16] R. Lyons and Y. Peres. Probability on trees and networks, volume 42 of Cambridge Series in Statistical and Probabilistic Mathematics. Cambridge University Press, New York, 2016. MR3616205

[LSW04] G. F. Lawler, O. Schramm, and W. Werner. Conformal invariance of planar looperased random walks and uniform spanning trees. Ann. Probab., 32(1B):939-995, 2004. MR2044671

[LSW19] G. F. Lawler, X. Sun, and W. Wu. Four dimensional loop-erased random walk. Ann. Probab., 2019. To appear.

[McG91] S. McGuinness. Recurrent networks and a theorem of Nash-Williams. J. Theoret. Probab., 4(1):87-100, 1991. MR1088394

[Mor03] B. Morris. The components of the wired spanning forest are recurrent. Probab. Theory Related Fields, 125(2):259-265, 2003. MR1961344

[NW59] C. St. J. A. Nash-Williams. Random walk and electric currents in networks. Proc. Cambridge Philos. Soc., 55:181-194, 1959. MR23:A2239

[Pem91] R. Pemantle. Choosing a spanning tree for the integer lattice uniformly. Ann. Probab., 19(4):1559-1574, 1991. MR1127715 (92g:60014)

[Pet83] K. Petersen. Ergodic theory, volume 2 of Cambridge Studies in Advanced Mathematics. Cambridge University Press, Cambridge, 1983. MR833286

[Sch00] O. Schramm. Scaling limits of loop-erased random walks and uniform spanning trees. Israel J. Math., 118:221-288, 2000. MR1776084

[She16] S. Sheffield. Quantum gravity and inventory accumulation. Ann. Probab., 44(6):3804-3848, 2016. MR3572324

[Sun19] X. Sun. Random planar geometry through the lens of uniform spanning tree. Bernoulli News, 26(2):10-13, 2019. 
[Wil96] D. B. Wilson. Generating random spanning trees more quickly than the cover time. In Proceedings of the Twenty-Eighth Annual ACM Symposium on the Theory of Computing (Philadelphia, PA, 1996), pages 296-303. ACM, New York, 1996. MR1427525 